\section{ORIGINAL} RESEARCH

B. Wu

X. Wang

J. Guo

S. Xie

E.C. Wong

J. Zhang

X. Jiang

J. Fang

\title{
Collateral Circulation Imaging: MR Perfusion Territory Arterial Spin-Labeling at 3T
}

\begin{abstract}
BACKGROUND AND PURPOSE: Current knowledge of the collateral circulation remains sparse, and a noninvasive method to better characterize the role of collaterals is desirable. The aim of our study was to investigate the presence and distal flow of collaterals by using a new MR perfusion territory imaging, vessel-encoded arterial spin-labeling (VE-ASL).
\end{abstract}

MATERIALS AND METHODS: Fifty-six patients with internal carotid artery (ICA) or middle cerebral artery (MCA) stenosis were identified by sonography. VE-ASL was performed to assess the presence and function of collateral flow. The perfusion information was combined with VE maps into high signalintensity-to-noise-ratio 3-colored maps of the left carotid, right carotid, and posterior circulation territories. The presence of the anterior and posterior collateral flow was demonstrated by the color of the standard anterior cerebral artery/MCA flow territory. The distal function of collateral flow was categorized as adequate (cerebral blood flow $[C B F] \geq 10 \mathrm{~mL} / \mathrm{min} / 100 \mathrm{~g}$ ) or deficient (CBF $<10 \mathrm{~mL} / \mathrm{min} / 100 \mathrm{~g}$ ). The results were compared with those of MR angiography (MRA) and intra-arterial digital subtraction angiography (DSA) in cross table, and $\kappa$ coefficients were calculated to determine the agreement among different methods.

RESULTS: The $\kappa$ coefficients of the presence of anterior and posterior collaterals by using VE-ASL and MRA were 0.785 and 0.700 , respectively. The $\kappa$ coefficient of the function of collaterals by using VE-ASL and DSA was 0.726 . Apart from collaterals through the circle of Willis, VE-ASL showed collateral flow via leptomeningeal anastomoses.

CONCLUSIONS: In patients with ICA or MCA stenosis, VE-ASL could show the presence, the origin, and distal function of collateral flow noninvasively.

$\mathbf{T}$ he protective effect of collateral circulation influences final clinical outcomes for patients with hemodynamically significant arterial stenosis. The principal source of collateral flow of cerebral arteries is through the arteries of the circle of Willis. Secondary collateral pathways include the external carotid artery via the ophthalmic artery and leptomeningeal anastomoses at the brain surface. However, the size and patency of these arteries are quite variable.

Doppler sonography is the most common tool used to investigate the presence of collateral flows. MR angiography (MRA) can be used for determining the collaterals through the circle of Willis. However, both sonography and MRA do not show leptomeningeal collateral pathways, distal collateral flows, or the actual contribution of collateral flow to brain perfusion. Intra-arterial digital subtraction angiography (DSA) shows the presence and distal arteries of the collateral pathways. ${ }^{1}$ However, to visualize all the collateral pathways, this technique requires an invasive and selective 3-vessel approach and is typically not performed in patients with acute stroke or cerebral arterial stenosis. Therefore, a noninvasive method that demonstrates selectively angiographic information may be desired to investigate collateral blood flow. ${ }^{2}$

In MR perfusion territory arterial spin-labeling (ASL), ${ }^{3-7}$ blood in individual or groups of feeding arteries is tagged by

Received May 2, 2008; accepted after revision June 25.

From the Department of Radiology (B.W., X.W., S.X., X.J.), Peking University First Hospital, Beijing, China; Interdisciplinary Academy (B.W., X.W., J.G., J.Z., J.F.), Peking University, Beijing, China; and Center for Functional Magnetic Resonance Imaging and Departments of Radiology and Psychiatry (E.C.W.), University of California, San Diego, La Jolla, Calif.

Please address correspondence to Xiaoying Wang, MD, Department of Radiology, Peking University First Hospital, Xishiku Rd, Xicheng District, Beijing, 100034, China; e-mail: cjr.wangxiaoying@vip.163.com

DOI 10.3174/ajnr.A1259 using ASL methodology, and images are acquired that map the vascular distribution of those feeding arteries. Recently, vessel-encoded ASL (VE-ASL) MR imaging ${ }^{8}$ was introduced as a more time-efficient method for mapping multiple vascular territories. The aim of our study was to investigate the presence and distal flow of collateral blood supply by using the VE-ASL technique at $3 \mathrm{~T}$ on patients with carotid stenotic disease.

\section{Materials and Methods}

\section{Patients}

Fifty-six consecutive patients (30 men, 26 women; mean age, 58.0 years; range, 33-82 years) were referred to the department of radiology by vascular surgeons or neurologists for diagnosis and grading of internal carotid artery (ICA) or middle cerebral artery (MCA) severe stenosis or occlusion between July and December 2007. All patients were identified with evidence of $>70 \%$ ICA stenosis by color-coded sonography ${ }^{9}$ or MCA stenosis (or occlusion) by transcranial Doppler (TCD) sonography. ${ }^{10}$

Fifty-one patients had hemispheric transient ischemic attack $(n=$ 34 ) or other minor deficits $(n=17)$, including weakness on 1 side of the body, reduced vision, or dizziness within 1 month before the MR imaging examination. Five patients with unilateral asymptomatic ICA occlusion were included ( 3 men, 2 women). Their carotid artery occlusions were detected in a general screening. The ethics committee approved the study protocol, and written informed consent was obtained from all participants.

\section{MR Imaging}

MR investigations were performed with a 3T whole-body system (High Definition; GE Healthcare, Milwaukee, Wis) by using a com- 

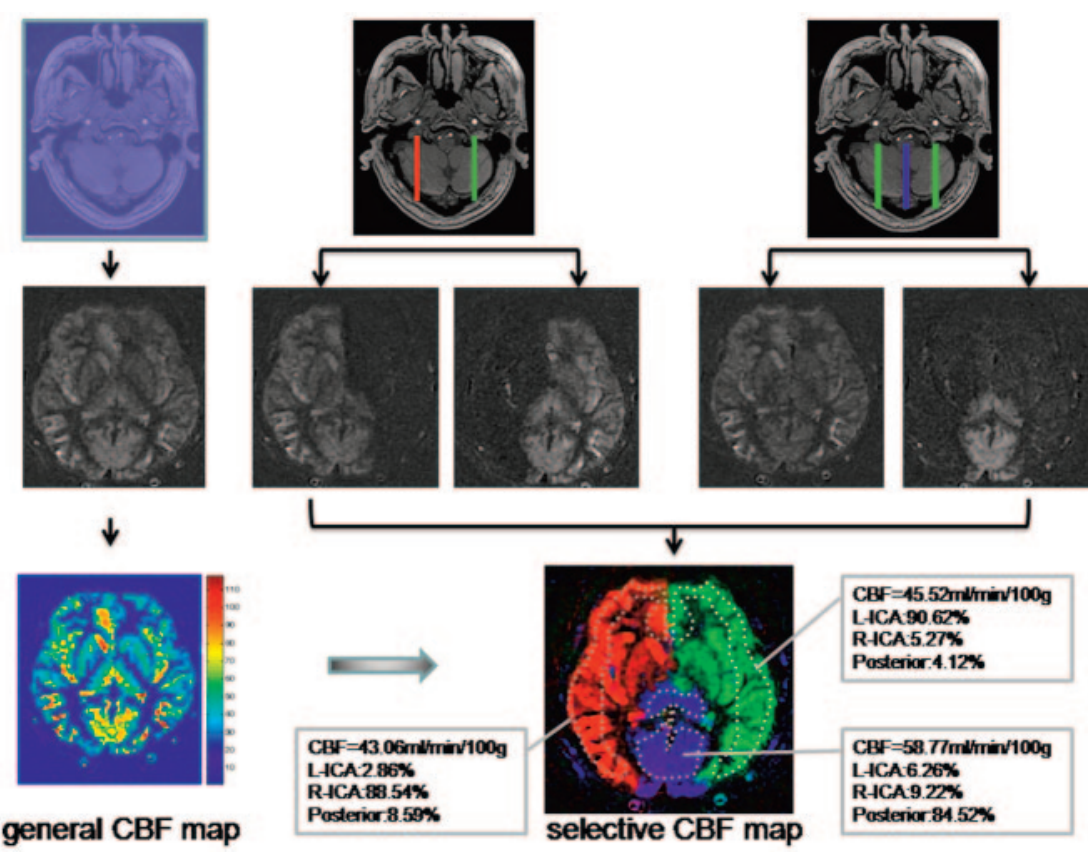

Fig 1. VE processing steps in a volunteer. Top, Positioning and orientation of the labeling area on a section of TOF MRA dataset. The labeling area is shown in the first image as the whole section, in the second image as the right (R) and left (L) ICA, and in the third image as ICAs and posterior circulation. Middle, Raw CBF map and reconstruction of separated vascular territories. Bottom, 3-Color selective CBF map is constructed by a combination of the general CBF map with the vascular territory map. Examples of regions of interest present the CBF values and percentages of left, right ICA, and posterior circulation.

mercial 8-channel head radio-frequency coil array and body coil for radio-frequency transmission.

\section{$V E-A S L$}

VE-ASL perfusion imaging was performed by using a selective perfusion imaging sequence, which was based on pseudocontinuous ASL. ${ }^{11}$ To encode all vessels of interest, we modified a pseudocontinuous tagging pulse train by using additional transverse gradient pulses and phase cycling to place some arteries in a tag condition, while others passing through the same tagging plane were in a control condition. ${ }^{8}$

Seven axial imaging sections were acquired in the caudal-to-cranial direction in 6 minutes. For image acquisition, a 4-shot spiral readout was used. The tagging pulse-train length was $1600 \mathrm{~ms}$. The postlabeling delay time was set at $1000 \mathrm{~ms}$. Other MR imaging parameters were as follows: TR/TE, 3000/3.4 ms; section thickness/section gap, 8/2 mm; FOV, 240 mm; matrix, 128.

Data were processed with Matlab (MathWorks, Natick, Mass). The artery selectivity of this method was demonstrated by using 3 different color maps. The cerebral blood flow (CBF) images were obtained by subtraction of the labeled from the control images. To quantify CBF, perfusion-weighted ASL signal intensity was fitted to the perfusion model described by Wong ${ }^{12}$ with the following values for the physical constants: $\alpha$ (efficiency of the inversion pulse) $=0.7, \mathrm{~T}_{1 \text { blood }}=1664 \mathrm{~ms}^{13}$ The perfusion information was combined with VE maps into high signalintensity-to-noise-ratio 3-colored selective CBF maps of the left carotid, right carotid, and posterior circulation territories (Fig 1).

In our study, "anterior collateral flow" was defined as retrograde flow (abnormal flow) in the A1 segment on the steno-occluded side that supplied the A2 segment and the MCA on the side of the stenoocclusion. "Posterior collateral flow" was defined as antegrade flow (abnormal flow) in the posterior communicating artery (PcomA).

The presence of the anterior and posterior collateral flow was demonstrated by the color of the standard anterior cerebral artery
(ACA)/MCA flow territory ipsilateral to the stenotic side. To compare with 2D phase-contrast MRA (2D-PC MRA), we categorized flow patterns of the A1 segment and PcomA as follows: 1) no flow, 2) normal flow, and 3) abnormal flow (Fig 2). "Normal" meant antegrade $\mathrm{A} 1$ and retrograde PcomA.

The distal collateral flow of the stenotic hemisphere, including standard ACA/MCA flow territory ${ }^{14}$ and the basal nuclei area, was recorded separately as adequate $(\mathrm{CBF} \geq 10 \mathrm{~mL} / \mathrm{min} / 100 \mathrm{~g})$ or deficient $(\mathrm{CBF}<10$ $\mathrm{mL} / \mathrm{min} / 100 \mathrm{~g}$ ). We hypothesized that adequate blood perfusion represents adequate distal flow of the collateral circulation. To eliminate the areas of brain infarction, we carefully excluded large high-signal-intensity lesions on T2-weighted imaging if present.

\section{D Time of Flight and 2D-PC MRA}

The circle of Willis was visualized with a 3D time-of-flight (3D-TOF) MRA sequence (TR, $21 \mathrm{~ms}$; TE, $3.2 \mathrm{~ms}$, flip angle, $15^{\circ}$; 1 signal acquired; 176 sections; section thickness, $1.6 \mathrm{~mm}$ with a section overlap of $0.8 \mathrm{~mm}$; FOV, $240 \mathrm{~mm}$; matrix size, 384; acquisition time, $5 \mathrm{~min}$ utes), after which a reconstruction was made in 3 orthogonal directions with a maximum intensity projection algorithm.

The direction of blood flow in the circle of Willis was assessed with 2 consecutive 2D-PC measurements, of which 1 was phase-encoded in the anteroposterior direction and the second, in the left-right direction (TR, $18 \mathrm{~ms}$; TE, $6.2 \mathrm{~ms}$; flip angle, $8.0^{\circ}$; section thickness, 13 mm; 4 averages; FOV, 250 mm; matrix, 256; velocity sensitivity, 40 $\mathrm{cm} / \mathrm{s}$; acquisition time, 37 seconds per direction; no cardiac gating). Previous studies have indicated that PC MRA provides a reliable method to assess the direction of flow in the circle of Willis. ${ }^{15-18}$

In each patient, the presence of the anterior and posterior collateral flow was demonstrated by flow patterns of the A1 segment and PcomA. The patterns were categorized as follows: 1) no flow, 2) normal flow, and 3) abnormal flow. 


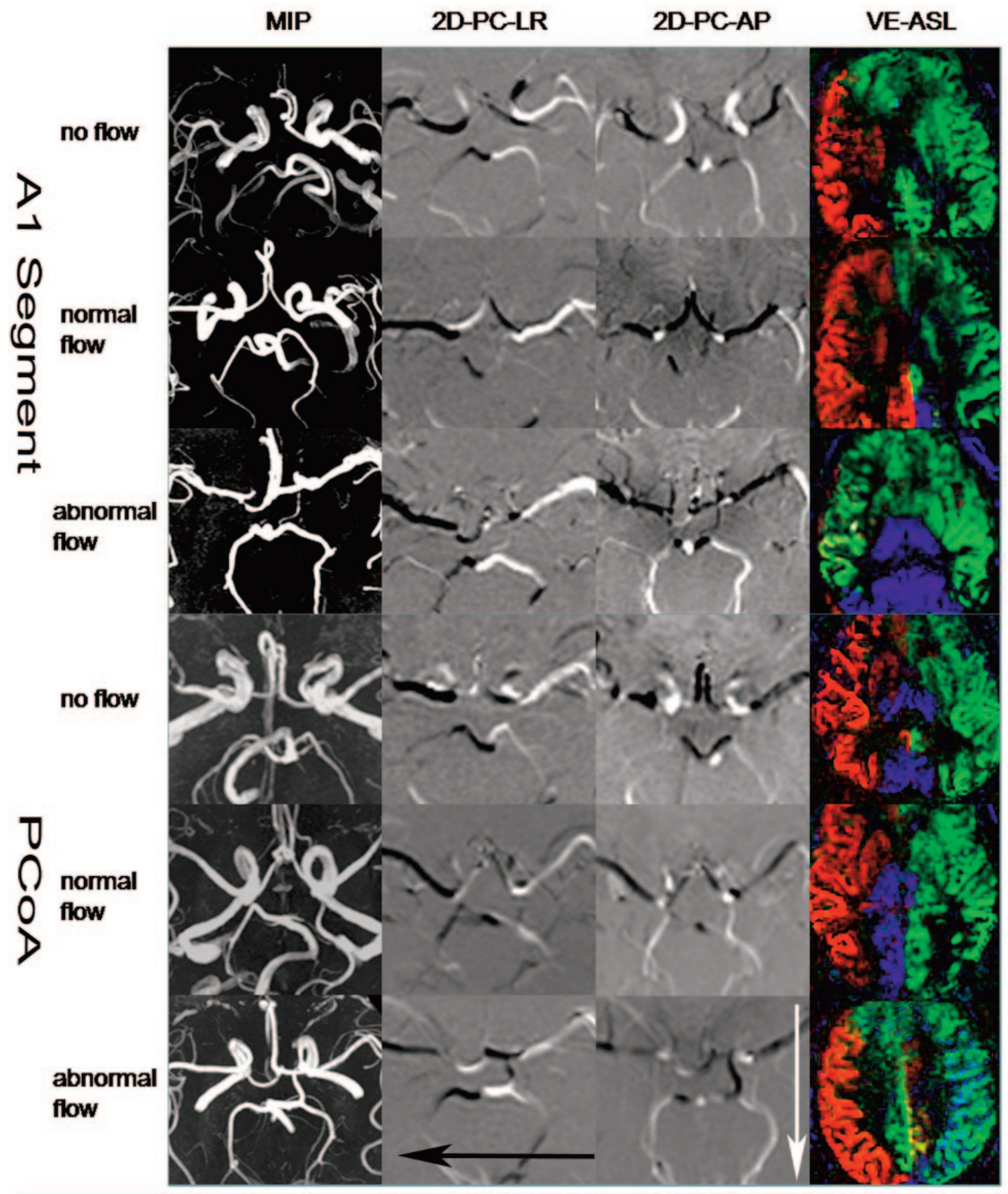

Fig 2. The A1 segment and PcomA flow pattern on MRA and VE-ASL investigations in 6 patients with ICA stenosis. Each row of images represents a patient. Column 1, Maximum intensity projections (MIPs) of the circle of Willis as investigated with 3D MRA. Column 2, 2D-PC images that are phase-encoded in the left-right (LR) direction. Black arrow shows the direction of the black color. Column 3, 2D-PC images that are phase-encoded in the anteroposterior (AP) direction. White arrow shows the direction of the white color. Column 4, VE-ASL images. Red, green, and blue indicate the right ICA, left ICA, and posterior circulation, respectively.

\section{DSA}

Symptomatic patients with $>70 \%$ ICA obstruction on sonography or MCA occlusion on TCD were recommend for angiography. One experienced neuroradiologist, who was blinded to all clinical information, reviewed the angiograms. In each DSA study, the distal flow ipsilateral to the side of $>70 \%$ ICA or MCA severe stenosis was ob- served following a carotid or vertebral injection. Distal collateral flow of standard ACA/MCA territories and basal nuclei (lenticulostriate artery) was recognized as adequate by the contrast filling the small arteries of the corresponding areas. Distal collaterals were considered deficient if they were not visualized on the angiogram. To grade the appearance of distal collaterals, the neuroradiologist determined an- 


\begin{tabular}{|c|c|c|c|c|}
\hline \multicolumn{5}{|c|}{$\begin{array}{l}\text { Table 1: Cross table of the A1 segment and PcomA flow on MRA } \\
\text { and VE-ASL }\end{array}$} \\
\hline \multirow[b]{2}{*}{ MRA } & \multicolumn{3}{|c|}{ VE-ASL } & \multirow[b]{2}{*}{ Total } \\
\hline & No Flow & $\begin{array}{l}\text { Normal } \\
\text { Flow }\end{array}$ & $\begin{array}{l}\text { Abnormal } \\
\text { Flow }\end{array}$ & \\
\hline \multicolumn{5}{|l|}{ A1 segment } \\
\hline No flow & $16(28.6 \%)$ & $0(0.0 \%)$ & $0(0.0 \%)$ & $16(28.6 \%)$ \\
\hline Normal flow & $6(10.7 \%)$ & $32(57.1 \%)$ & $0(0.0 \%)$ & $38(67.9 \%)$ \\
\hline Abnormal flow & $0(0.0 \%)$ & $0(0.0 \%)$ & $2(3.6 \%)$ & $2(3.6 \%)$ \\
\hline Total & $22(39.3 \%)$ & $32(57.1 \%)$ & $2(3.6 \%)$ & $56(100.0 \%)$ \\
\hline \multicolumn{5}{|l|}{ PcomA } \\
\hline No flow & $24(42.9 \%)$ & $4(7.1 \%)$ & $3(5.4 \%)$ & 31 (55.4\%) \\
\hline Normal flow & $3(5.4 \%)$ & $16(28.6 \%)$ & $0(0.0 \%)$ & $19(33.9 \%)$ \\
\hline Abnormal flow & $0(0.0 \%)$ & $0(0.0 \%)$ & $6(10.7 \%)$ & $6(10.7 \%)$ \\
\hline Total & $27(48.2 \%)$ & $20(35.7 \%)$ & $9(16.1 \%)$ & $56(100.0 \%)$ \\
\hline
\end{tabular}

Note:-PcomA indicates posterior communicating artery; MRA, MR angiography; VE-ASL, vessel-encoded arterial spin-labeling.

Table 2: Cross table of the distal flow of the hemisphere of the stenotic side on intra-arterial DSA and VE-ASL

\begin{tabular}{llcc}
\hline & \multicolumn{2}{c}{ VE-ASL } & \\
\cline { 2 - 3 } DSA & Deficiency & Adequacy & Total \\
\hline Deficiency & $5(12.8 \%)$ & $0(0.0 \%)$ & $5(12.8 \%)$ \\
Adequacy & $3(7.7 \%)$ & $31(79.5 \%)$ & $34(87.2 \%)$ \\
Total & $8(20.5 \%)$ & $31(79.5 \%)$ & $39(100.0 \%)$ \\
\hline
\end{tabular}

Note:-DSA indicates digital subtraction angiography.

giographic collateral degrees ( $0-3)$, in which 0 indicated no filling by contrast medium of the distal vessel through the collaterals; 1 indicated slight collateral distribution, often with dilution; 2 indicated small but definite collateral supply; and 3 indicated the main vessel and distal vessel filled through the collaterals. For the purpose of the present study, grades 2 and 3 were combined to indicate the adequate supply of collateral pathways. Collaterals were considered deficient if they were assigned grades 0 or 1 . To assess the distal flow of collateral pathways, the observer examined biplane (anteroposterior, lateral, and/or oblique) views. For each projection, $6-8 \mathrm{~mL}$ of contrast material (Ultravist [300 mg of iodine per milliliter]; Schering, Berlin, Germany) was injected at a flow rate of $4 \mathrm{~mL} / \mathrm{s}$.

\section{Statistical Analysis}

Cross table was used to compare VE-ASL and MRA for determination of the patterns of collateral flow based on the circle of Willis by calculating $\kappa$ coefficients. For the patients with unilateral ICA or MCA obstruction, cross table was also used to compare VE-ASL and DSA on imaging the distal flow of collateral blood based on the 3 major cerebral feeding arteries. DSA was considered the gold standard method.

\section{Results}

All patients were studied with VE-ASL and MRA. Of all involved patients, 15 were studied with selective angiography. Of those patients, 5 had unilateral ICA occlusion, 7 had unilateral ICA stenosis (>90\% obstruction), 1 had unilateral MCA occlusion, and the other 2 had Moyamoya disease.

\section{Collateral Flow via the Circle of Willis on VE-ASL and MRA}

The prevalence in the A1 segment of no flow, normal flow, and abnormal flow by using MRA was $28.6 \%, 67.9 \%$, and $3.6 \%$, respectively, for all patients. The prevalence as measured by
VE-ASL was $39.3 \%, 57.1 \%$, and $3.6 \%$, correspondingly. The measure of agreement $(\kappa)$ was $0.785(P<.01)$. The prevalence in the PcomA of no flow, normal flow, and abnormal flow by using MRA was 55.4\%, 33.9\%, and $10.7 \%$, respectively, for those patients. The prevalence measured by using VE-ASL was $48.2 \%, 35.7 \%$, and $16.1 \%$, respectively. The $\kappa$ coefficient was $0.700(P<.01)$ (Table 1$)$.

In $6(10.7 \%)$ patients, VE-ASL showed that the ACA flow territory was supplied mainly by the contralateral ACA, whereas MRA showed the presence of ACA flow. In 3 patients, the diameter of the stenotic side ACA was much thinner than that of the contralateral side. In the other 3 patients, the diameter of bilateral ACAs was almost equal.

In $4(7.1 \%)$ patients, VE-ASL showed that the stenotic ICA supplied the posterior circulation flow territory, whereas MRA did not show the presence of the PcomA. Moreover, in 3 (5.4\%) patients, VE-ASL revealed that posterior circulation supplied the MCA flow territory, but MRA illustrated the absence of the PcomA.

\section{Distal Collateral Flow on VE-ASL and DSA}

We observed 39 brain areas of 13 hemispheres ipsilateral to the side of unilateral severe stenosis in 13 patients with unilateral obstruction. The prevalence of adequate and deficient flow was $87.2 \%$ (34) and $12.8 \%$ (5) by using DSA and 79.5\% (31) and $20.5 \%$ (8) by using VE-ASL. The $\kappa$ coefficient was 0.726 $(P<.01)$ (Table 2$)$.

Patients with Unilateral ICA Severe Stenosis. In 6 patients, VE-ASL showed that collateral flow from the posterior circulation supplied most of the stenotic hemisphere. The contralateral ICA supplied the ACA flow territory of the stenotic side. Both of these results were in agreement with DSA findings (Fig 3). In 4 patients, both VE-ASL and DSA showed the flow deficiency of the MCA flow territory. In 2 patients, the flow supply was difficult to determine by using VE-ASL, whereas DSA showed blood flow via the ophthalmic arteries that supplied the MCA. In 1 patient, the flow supply of the ACA area was difficult to determine by using VE-ASL, whereas DSA showed very slow leptomeningeal collaterals from the contralateral MCA supplying that area.

Patients with Bilateral ICA Severe Stenosis. Both VE-ASL and DSA showed the absence of the MCA with multiple collateral vessel perfusion from the posterior circulation. The bilateral basal nuclei areas were supplied by the stenotic ICA or posterior circulation in VE-ASL in accordance with findings on DSA.

\section{Discussion}

The potential of the circle of Willis to provide alternative flow routes in case of diminished arterial flow to the brain has been known since Sir Thomas Willis first described the collateral function of the arterial anastomoses in $1664 .{ }^{19}$ For patients with ICA stenosis or occlusion, both long-term and perioperatively, identification of collaterals assists in identifying patients with severe ICA stenosis at lower risk of stroke and transient ischemic attack. ${ }^{1}$ Still, the importance of the individual pathways in the circle itself is not always clear in any given patient. 3D-time of flight, 2D-PC MRA, and TCD may show the presence of collateral flow, but reliable quantitative flow measurements may not be achieved because of the small diameter or 


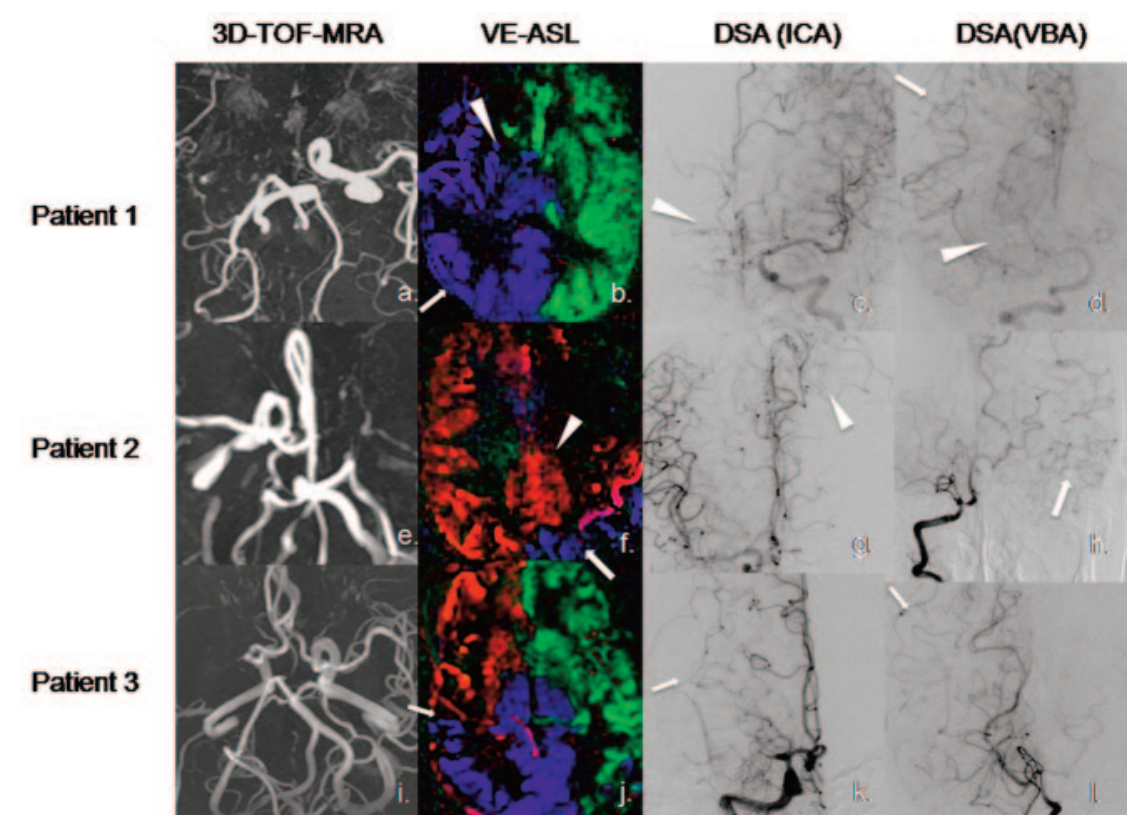

Fig 3. VE-ASL and intra-arterial DSA in 3 patients. Column 1, The circle of Willis as investigated with MRA, collapsed axial maximum intensity projection. Column 2, VE-ASL images. Column 3, DSA images following an ICA injection of contrast agent. Column 4, DSA images following a vertebrobasilar artery (VBA) injection of contrast agent. For the first patient, VE-ASL shows collaterals from the VBA supplying the right ICA territory (arrow in a). The right basal nuclei is supplied by the VBA (blue) and the contralateral ICA (green) in VE-ASL (arrowhead). In the second patient, the left ACA/MCA flow territory is supplied by the contralateral ACA (arrowhead) and the VBA (arrows in $f$ and $I$ ). The right MCA flow territory of the third patient is supplied by the ipsilateral ACA and VBA (arrow).

tortuosity of involved arteries. Xenon CT, single-photon emission CT, positron-emission tomography, CT perfusion, and MR perfusion provide information regarding the amount of blood flow to specific regions of the brain, though the arterial source of sustained perfusion may not be evident when the parent vessel is occluded.

VE-ASL introduces perfusion information from 3 major cerebral feeding vessels, which could reveal the presence of collateral vessels and the contribution of such flow to brain perfusion. In our study, VE-ASL had good agreement with 2D-PC MRA on evaluating collaterals through the circle of Willis. Evaluating distal collateral flow is the crucial advantage over MRA. Compared with other perfusion techniques, the 3-color selective perfusion map displayed the blood territory from 3 major feeding vessels, making it simple to show the evidence of severe stenosis or occlusion of the parent vessels. Our study also showed that the results of VE-ASL had a good agreement with the distal collaterals on $\mathrm{x}$-ray angiography (Fig 3). To our knowledge, this is the first study to compare the selective ASL MR perfusion with DSA for determination of the presence and distal function of collateral circulation.

We also found results similar to those of van Laar et $\mathrm{al}^{3}$ in the few patients with carotid occlusion whom we enrolled. In patients with ICA occlusion, the MCA flow territory ipsilateral to the occluded ICA is mainly supplied by the vertebrobasilar arteries, whereas the ACA flow territory on the occluded side is mainly supplied by the contralateral ICA. ${ }^{3}$

The discrepancy between MRA and VE-ASL was related to determination of the antegrade flow of the A1 segment and the presence of the PcomA. Two patients with conflicting results of the Al segment were studied with selective angiography, which proved that the ACA flow territory was supplied mainly by the contralateral ACA. Decreased perfusion pressure might result in a decrease in supply of the ipsilateral ACA to the stenotic side. As to the PcomA, 4 patients had stenotic ICA supplying the posterior circulation and 3 patients had posterior circulation supplying the MCA flow territory, despite the absence of a PcomA on MRA. This finding implies that additional information beyond that supplied by the circle of Willis is being picked up by the ASL method. DSA of these patients also showed that the major reason for disagreement was collaterals through leptomeningeal anastomoses, as shown on the first and second patients in Fig 3. VE-ASL showed collateral flow from the posterior circulation via leptomeningeal anastomoses, which were another important route of collateral circulation when the PcomA was absent. Robust leptomeningeal collaterals have been linked to rapid recanalization of MCA occlusion and possible prevention of larger infarcts. ${ }^{20}$ The difference between the results by using DSA and VE-ASL was related to collaterals via the ophthalmic artery or the long transit time of collaterals from contralateral leptomeningeal anastomoses.

The present study may have several limitations. First, selective ASL was performed at a single delay time between labeling and imaging. In general, this delay time is sufficient for adequate delivery of labeled blood to the brain for healthy volunteers. However, with severe obstruction of the main feeding arteries and delayed perfusion by the leptomeningeal collateral circulation, the arrival time of the labeled blood at the brain may be increased. This may result in an underestimation of the flow territory or the amount of perfusion, and a long transit time also limits the applicability of ASL for measurement of perfusion in white matter. ${ }^{21}$ Therefore, the threshold for assessing distal collateral flow we chose was lower than that reported in the literature. ${ }^{22}$

The second limitation is that the imaging technique used in our study does not take into account flow from the ipsilateral external carotid artery, though the methodology can, in prin- 
ciple, do so. In previous studies, researchers found that nearly all patients with bilateral ICA occlusion had retrograde flow in the ophthalmic artery. ${ }^{18}$ Another limitation may be that though conventional angiography is considered the gold standard, objective evaluation of collaterals is rarely performed. Variation in contrast volume and pressure during injection may distort the appearance of distal vessels. ${ }^{2}$ Moreover, we studied only 15 patients with angiography. The number of patients is too small to obtain definite conclusions about the utility of VE-ASL. Moreover, stenotic lesions of cerebral vessels other than ICA/MCA and anatomic abnormalities were not considered in the present study.

\section{Conclusions}

The present study provided preliminary evidence that VE-ASL could show the presence and distal flow of collaterals in patients with ICA steno-occlusion.

\section{Appendix}

\section{Theory}

The model for the pseudocontinuous ASL (PCASL) calculation is the same as that in the model for continuous ASL $(\mathrm{CASL})^{12,23}$ :

$C B F=$

$\frac{\operatorname{Sig}_{A S L}}{2 \cdot \alpha \cdot R \cdot \operatorname{Sig}_{C S F} \cdot T_{1 B} \cdot\left(\exp \left(-(v t i-p w p c a s l) / T_{1 B}\right)-\exp \left(-v t i / T_{1 B}\right)\right)}$,

where $\operatorname{Sig}_{A S L}$ is the ASL signal intensity, $S i g_{A S L}=S i g_{\text {con }}-S i g_{\text {tag }}, \alpha$ is the tagging efficiency of PCASL, $R$ is the signal-intensity ratio between fully relaxed arterial blood and CSF, Sig ${ }_{C S F}$ is the signal intensity of the CSF that we picked from the region of the cerebral lateral ventricle, $T_{1 \mathrm{~B}}=1.664 \mathrm{~s}^{13}$ and is the T1 relaxation time of arterial blood on 3T, $v t i$ is the time between the beginning of the tag pulse and the excitation pulse of the imaging part (TI time) for different sections. The delay time (sldelay) between sections was considered because we used the continuous acquisition order: $v t i(i)=v t i(1)+(i-1)^{*}$ sldelay, where $v t i(i)$ is the TI time of the $i$ section ( $\mathrm{i}$ is the number of the section), and pwpcasl is the tagging duration of PCASL.

\section{The Postprocessing Scheme}

1) Use a region-growing strategy to generate the brain mask.

2) Use the minimum of contrast images (which are in another acquisition) to estimate the B1 sensitivity of the coil and correct the acquired signal intensity.

3) Calculate the $\operatorname{Sig}_{A S L}=S i g_{c o n}-S i g_{\text {tag }}$, by using a simple subtraction method and average them throughout the acquisition points.

4) Pick the region of the ventricle and choose the pixel with the strongest signal intensity because the voxel that is fully occupied by CSF will give the strongest signal intensity with the following imaging parameters: $\mathrm{TR}=6 \mathrm{~s}$, and $\mathrm{TE}=3.1 \mathrm{~ms}$, flip angle $=90^{\circ}$, which is proton-attenuation-like contrast, thus we got the Sig $_{C S F}$;

5) The $\alpha$ we used is 0.7 , which is derived from our measurements with the same pulse sequence parameters on volunteers and the $R$ we used is 0.93 .

\section{References}

1. Henderson RD, Eliasziw M, Fox AJ, et al. Angiographically defined collateral circulation and risk of stroke in patients with severe carotid artery stenosis. Stroke 2000;31:128-32

2. Liebeskind DS. Collateral circulation. Stroke 2003;34:2279-84

3. van Laar PJ, Hendrikse J, Klijn CJ, et al. Symptomatic carotid artery occlusion flow territories of major brain-feeding arteries. Radiology 2007;242:526-34

4. Lim CC, Petersen ET, Ng I, et al. MR regional perfusion imaging: visualizing functional collateral circulation. AJNR Am J Neuroradiol 2007;28:447-48

5. van Osch MJ, Hendrikse J, Golay X, et al. Non-invasive visualization of collateral blood flow patterns of the circle of Willis by dynamic MR angiography. Med Image Anal 2006;10:59-70. Epub 2005 Jun 13

6. van Laar PJ, Hendrikse J, Golay X, et al. In vivo flow territory mapping of major brain feeding arteries. Neuroimage 2006;29:136-44

7. Hendrikse J, van der Grond J, Lu H, et al. Flow territory mapping of the cerebral arteries with regional perfusion MRI. Stroke 2004;35:882-87

8. Wong EC. Vessel-encoded arterial spin labeling using pseudocontinuous tagging. Magn Reson Med 2007;58:1086-91

9. Grant EG, Benson CB, Moneta GL, et al. Carotid artery stenosis: gray-scale and Doppler US diagnosis-Society of Radiologists in Ultrasound Consensus Conference. Radiology 2003;229:340-46

10. Bang OY, Cho JH, Han BI, et al. Transcranial Doppler findings in middle cerebral arterial occlusive disease in relation to degree of stenosis and presence of concomitant stenoses. J Clin Ultrasound 2003;31:142-51

11. Garcia DM, de Bazelaire C, Alsop D. Pseudo-Continuous Flow-Driven Adiabatic Inversion for Arterial Spin Labeling: Proceedings of the International Society for Magnetic Resonance in Medicine, Miami, Fla, 7-13 May 2005. Miami: John Wiley \& Sons; 2005:37

12. Wong EC. Quantifying CBF with pulsed ASL: technical and pulse sequence factors. J Magn Reson Imaging 2005;22:727-31

13. Lu H, Clingman C, Golay X, et al. Determining the longitudinal relaxation time (T1) of blood at 3.0 Tesla. Magn Reson Med 2004;52:679-82

14. van der Zwan A, Hillen B, Tulleken CA, et al. Variability of the territories of the major cerebral arteries. J Neurosurg 1992;77:927-40

15. Miralles M, Dolz JL, Cotillas J, et al. The role of the circle of Willis in carotid occlusion: assessment with phase contrast MR angiography and transcranial duplex. Eur J Vasc Endovasc Surg 1995;10:424-30

16. Ross MR, Pelc NJ, Enzmann DR. Qualitative phase contrast MRA in the normal and abnormal circle of Willis. AJNR Am J Neuroradiol 1993;14:19-25

17. Pernicone JR, Siebert JE, Laird TA, et al. Determination of blood flow direction using velocity-phase image display with 3-D phase-contrast MR angiography. AJNR Am J Neuroradiol 1992;13:1435-38

18. Rutgers DR, Klijn CJ, Kappelle LJ, et al. A longitudinal study of collateral flow patterns in the circle of Willis and the ophthalmic artery in patients with a symptomatic internal carotid artery occlusion. Stroke 2000;31:1913-20

19. Eastcott HH. The beginning of stroke prevention by surgery. Cardiovasc Surg 1994;2:164-69

20. Ringelstein EB, Biniek R, Weiller C, et al. Type and extent of hemispheric brain infarctions and clinical outcome in early and delayed middle cerebral artery recanalization. Neurology 1992;42:289-98

21. van Gelderen P, de Zwart JA, Duyn JH. Pitfalls of MRI measurement of white matter perfusion based on arterial spin labeling. Magn Reson Med 2008;59:788-95

22. Bandera E, Botteri M, Minelli C, et al. Cerebral blood flow threshold of ischemic penumbra and infarct core in acute ischemic stroke: a systematic review. Stroke 2006;37:1334-39

23. Buxton RB, Frank LR, Wong EC, et al. A general kinetic model for quantitative perfusion imaging with arterial spin-labeling. Magn Reson Med 1998;40:383-96 